\title{
ANÁLISE DE REDES SOCIAIS NO ESPORTE PELA ÓTICA DA ABORDAGEM \\ ECOLÓGICA: UM ENSAIO TEÓRICO
}

\author{
SOCIAL NETWORKS ANALYSIS IN SPORTS FROM THE POINT OF VIEW OF THE \\ ECOLOGICAL APPROACH: A THEORETICAL FRAMEWORK
}

\section{ANÁLISIS DE REDES SOCIALES EN EL DEPORTE DESDE EL PUNTO DE VISTA DEL ENFOQUE ECOLÓGICO}

\begin{abstract}
Bruno Melli Neto ${ }^{1}$, Enrico Fuini Puggina ${ }^{1}$, Rafael Pombo Menezes ${ }^{1}$, João Cláudio Machado², Ricardo Vanella ${ }^{3}$, Rodrigo Aquino ${ }^{4}$

bruno.neto@usp.br; enrico@usp.br; rafaelpombo@usp.br; joaoclaudiomachado@gmail.com;
\end{abstract} vanella.ricardo.10@gmail.com; aquino.rlq@gmail.com

${ }^{1}$ Universidade de São Paulo, Ribeirão Preto, Brasil

2 Universidade Federal do Amazonas, Manaus, Amazonas

${ }^{3}$ Universidade do Porto, Porto, Portugal

${ }^{4}$ Universidade Federal do Espírito Santo, Vitória, Brasil

Envío original: 2021-02-20 Reenviado: 2021-06-14, 2021-08-08 Aceptado: 2021-08-10

Publicado: 2021-09-23

Doi: https://doi.org/10.15517/pensarmov.v19i2.45918

\begin{abstract}
RESUMO
A análise de desempenho é uma disciplina intimamente relacionada à pedagogia do esporte. As bases teóricas que sustentam as escolhas metodológicas devem ser bem estabelecidas para existir uma convergência entre os instrumentos de avaliação e o processo de ensino e treinamento. Este ensaio teórico teve por objetivo apresentar e discutir a importância e representatividade da análise de redes sociais para avaliar o desempenho em esportes coletivos. O principal objetivo da análise de redes sociais é estudar a relação entre os jogadores para identificar possíveis causas e consequências dos eventos ocorridos durante a partida. Sendo assim, a análise de redes sociais trabalha de forma diferente das análises tradicionais, nas quais o principal foco está no sujeito, ou em análises notacionais que são as mais comumente utilizadas e que buscam a acumulação da frequência dos eventos ocorridos (e.g. gols marcados, posse de bola, zonas de finalização). Tal instrumento de avaliação, teoricamente posicionado numa abordagem ecológica, mostra-se eficaz para a identificação dos padrões de interação em
\end{abstract}


um grupo e o entendimento dos artifícios sociais que ajudam a compreender o desempenho em uma equipe. Assim, as equipes passam a ser analisadas como grupos sociais e não como sujeitos isolados. Neste ensaio, também expomos as principais aplicações práticas desse instrumento de avaliação em diferentes esportes coletivos, como o futebol, futsal, handebol, basquetebol e voleibol.

Palavras-chave: pedagogia do esporte, análise de desempenho, análise de jogo, esportes coletivos, esportes de invasão.

\begin{abstract}
Performance analysis is a discipline closely related to sport pedagogy. The theoretical bases that support the methodological choices must be well established to exist a convergence between the evaluation instruments and the teaching and training process. This theoretical essay aimed to present and discuss the importance and representativeness of social network analysis to evaluate performance in team sports. The main objective of social network analysis is to study the relationship between players in order to identify possible causes and consequences of events that occur during a match. Thus, social network analysis works differently from traditional analyses, in which the main focus is on the subject, or on notational analyses, which are the most commonly used and seek to accumulate the frequency of events (e.g. goals scored, ball possession, finishing zones). Such an evaluation tool, theoretically positioned in an ecological approach, proves to be effective for the identification of interaction patterns in a group and the understanding of the social artifices that help understand a team's performance. Thus, teams come to be analyzed as social groups rather than as isolated subjects. In this essay, we also present the main practical applications of this evaluation tool in different team sports, such as soccer, futsal, handball, basketball, and volleyball.
\end{abstract}

Keywords: sport pedagogy, performance analysis, game analysis, team sports, invasion sports.

\title{
RESUMEN
}

El análisis del rendimiento es relacionado con la pedagogía del deporte. Las bases teóricas que sustentan las selecciones metodológicas deben estar bien establecidas para que haya una convergencia entre los instrumentos de evaluación y el proceso de enseñanza y entrenamiento. Este ensayo pretende presentar y discutir la importancia y representatividad del análisis de redes sociales para evaluar el rendimiento en los deportes de equipo. El objetivo principal del análisis de redes sociales es estudiar la relación entre los jugadores para identificar las posibles causas y consecuencias de los eventos durante el partido. Así, el análisis de redes sociales es diferente de los análisis tradicionales, en los que el foco principal está en el sujeto, o de los análisis notacionales, que son más utilizados y acumulan la frecuencia de los eventos ocurridos (por 
ejemplo, los goles marcados, la posesión del balón, las zonas de remate). Esta herramienta de evaluación, posicionada teóricamente en un enfoque ecológico, se muestra eficaz para la identificación de los patrones de interacción en un grupo y la comprensión de los artificios sociales que ayudan a entender el rendimiento de un equipo. Así, los equipos pasan a ser analizados como grupos sociales y no como sujetos aislados. En este ensayo, también exponemos las principales aplicaciones prácticas de esta herramienta de evaluación en diferentes deportes de equipo, como el fútbol, el fútbol sala, el balonmano, el baloncesto y el voleibol.

Palabras clave: pedagogía del deporte, análisis del rendimiento, análisis del juego, deportes de equipo, deportes de invasión.

\section{INTRODUÇÃO}

A pedagogia do esporte é um subcampo das Ciências do Esporte com grandes contribuições quanto à interface educativa presente nos esportes, tendo como objeto de estudo e intervenção o processo de ensino, vivência, aprendizagem e treinamento no esporte, buscando compreender como planejar, sistematizar, aplicar e avaliar este processo (Galatti et al., 2014). Durante todas as etapas do processo de ensino-aprendizagem dos esportes coletivos os/as professores/as e treinadores/as podem optar pela apropriação de métodos de ensino tradicionais (e.g. abordagem comportamental; tecnicismo) ou contemporâneos (e.g. processo pautado no jogo e centrado no jogador/a) (Scaglia et al., 2014, 2021).

A ruptura com o paradigma de ensino tecnocrático dos esportes coletivos constituída historicamente no Brasil e em outros países, por exemplo, emergiu da insatisfação com as limitações dos métodos tradicionais e culminou com a proposta de modelos contemporâneos de ensino (Menezes et al., 2014). Nesses, o conhecimento é entendido como resultante das interações da pessoa com o meio em que se está inserida, transitando de práticas esportivas centradas no treinador e pautadas na dimensão técnica, para práticas esportivas centradas no jogador e pautadas no jogo (Scaglia et al., 2014). Dentre esses, destaca-se a abordagem ecológica de ensino. A abordagem ecológica é um arcabouço teórico transdisciplinar integra conceitos da psicologia ecológica (Gibson, 1979), restrições sobre sistemas dinâmicos (Scott, 1995; Trevarthen et al., 1986), ciências da complexidade (Edelman \& Gally, 2001) e ciência evolucionária (Hatfield \& Kerick, 2007). Nessa abordagem teórica, conceitos como comportamento habilidoso, aprendizagem, expertise e talento são vistos como propriedades emergentes de uma relação funcionalmente adaptável, envolvendo relações entre o jogador (executor) e as restrições do ambiente e da tarefa (Araújo \& Davids, 2011). Ações habilidosas não são vistas como movimentos repetitivos do corpo fora do contexto, mas como interações dinâmicas, corpo-ambiente (Ingold, 2002), por meio das quais os indivíduos interagem para atingir os objetivos da tarefa (Woods et al., 2020). 
Tendo em vista a avaliação como um dos componentes da pedagogia do esporte, pode-se entender o processo de análise de desempenho, especialmente a análise de jogo, como um subcampo intimamente relacionado à pedagogia do esporte, quando seu propósito final é contribuir com o processo de ensino e treinamento e não uma análise meramente descritiva do jogo. A análise de jogo tem como propósito produzir conhecimento sobre o próprio jogo (oficial ou treinamento), seus jogadores e suas interações, compreendendo diferentes processos da avaliação (i.e. observação dos acontecimentos), por meio da coleta de dados e a interpretação dos mesmos (Garganta, 2001). Esse processo tem como consequência a busca de informações para subsidiar a tomada de decisão daqueles que buscam a melhora do desempenho esportivo (O'Donoghue, 2009), cuja objetividade, especificidade e representatividade das informações recolhidas são características importantes que podem ser influenciadas por diferentes instrumentos e métodos de análise (Carling et al., 2005; Groom et al., 2011).

De fato, percebemos diferentes métodos tradicionais e modelos contemporâneos para o ensino dos esportes coletivos que se relacionam diferentemente com o desenvolvimento das ações do jogo. Também podemos entender diferentes "caminhos" a serem percorridos no campo da análise de jogo, de modo que as intenções e interpretações produzidas pelas categorias de análise são oriundas de um arcabouço teórico dos profissionais e cientistas do esporte envolvidos nesse processo.

Teoricamente posicionada na abordagem ecológica, a análise de redes sociais destaca os processos interacionais estabelecidos pelos jogadores da equipe dentro e entre as equipes como o principal foco da análise de desempenho, fornecendo novas percepções sobre os processos sinérgicos subjacentes à organização e à função das equipes em ambientes de desempenho (Clemente, Martins, \& Mendes, 2015; Ribeiro et al., 2017). Este ensaio teórico tem por objetivo apresentar e discutir a importância e representatividade da análise de redes sociais para avaliar o desempenho em diferentes esportes coletivos.

\section{ABORDAGEM ECOLÓGICA}

Os esportes coletivos são representados como sistemas complexos e dinâmicos (Teodorescu \& Curado, 1984), nos quais as equipes são entendidas como um microsistema social, no qual o somatório das ações individuais não representa as relações de um grupo (Garganta, 1995). Ou seja, as ações realizadas por um jogador afetarão diretamente as ações de seus companheiros de equipe e seus oponentes (Menezes, 2012).

Durante o jogo, ocorre a constante disputa pela ordem por meio da desordem causada na equipe adversária, que por sua vez busca reestabelecer a ordem e reagir gerando a desordem nos seus adversários (Barreira, 2014). Tal processo ocorrerá constantemente durante toda a disputa, e a equipe que conseguir manter a ordem, ou ser capaz de gerir melhor a desordem, supostamente terá maiores chances de alcançar seus objetivos. 
Portanto, o processo de ensino-aprendizagem e treinamento deve estar alinhado à natureza estratégico-tática dos esportes coletivos, o que reafirma a importância dos modelos contemporâneos de ensino centrado do jogador e pautado no jogo (Machado et al., 2019), por manterem a complexidade inerente ao seu contexto (Menezes, 2012). Em contraste, ambientes de ensino e treino centrados no/a treinador/a e pautados em fragmentos de unidades (i.e. habilidade motoras básicas) e práticas reducionistas (i.e. fragmentação do jogo) não representam a natureza complexa dos esportes coletivos (Menezes, 2012).

A abordagem ecológica se baseia, portanto, nas relações espaciais e temporais dos indivíduos influenciadas por forças ambientais (Scaglia et al., 2014). Tal abordagem foi desenvolvida pelo psicólogo russo Urie Bronfenbrenner (Ettekal \& Mahoney, 2017) e explica como as pessoas sofrem e exercem influências de acordo com os ambientes em que estão inseridos, ou seja, como as relações mútuas pessoa-ambiente contribuem para as suas ações. A aprendizagem então se baseia na constante reciprocidade do sujeito e o meio em que se encontra inserido, bem como na tarefa a ser realizada, configurando a tríade sujeito-ambientetarefa.

Gibson (1979) propôs a partir desta abordagem que os indivíduos podem perceber as características do ambiente como possibilidades de ação (i.e. affordances) (Gibson, 1983), desde ambientes menores, com maior possibilidade de interação direta, até ambientes maiores, com maior possibilidade de interação indireta. Além disso, Bronfenbrenner propôs que a abordagem ecológica se baseia em quatro propriedades definidoras no desenvolvimento humano, sendo elas o indivíduo, o contexto, o processo e o tempo (Ettekal \& Mahoney, 2017).

No âmbito esportivo, esta abordagem vem sendo utilizada para investigar princípios que norteiam o desempenho esportivo e consequentemente o processo de interação, coordenação e tomada de decisão dos jogadores (Araújo \& Davids, 2009; Travassos et al., 2013; Vilar et al., 2012). Assim sendo, as relações entre os jogadores são dependentes das mudanças das condições que são impostas pelo contexto do jogo, o que supõe compreender que as ações de uma equipe não se instituem por meio da soma das ações individuais, mas nas interações entre companheiros para superar os adversários (Travassos et al., 2011). Arquiteta-se então o contínuo processo de adaptação mútua entre os jogadores para identificar as possibilidades de ação mais favoráveis de acordo com o espaço e tempo em que ocorrem os eventos na prática esportiva (Duarte et al., 2012; Passos et al., 2009; Travassos et al., 2012). Esse processo intencional e interativo envolvendo a percepção das possibilidades de ação que emergem durante o jogo leva o jogador a gerir melhor o espaço de jogo - "wayfinding", identificando e explorando seus muitos recursos (Silva et al., 2013; Woods et al., 2020).

Ao assumir o processo avaliativo como parte integrante da pedagogia do esporte (Galatti et al., 2014) pela ótica da abordagem ecológica considera três fatores: i) as interações dos jogadores; ii) o contexto e; iii) as decisões tomadas a partir das possibilidades de ação que 
emergem durante o período que será analisado. Dentre as variadas formas de considerar estes três aspectos durante o processo avaliativo (e.g. análise da coordenação das equipes por meio de dados posicionais (Low et al., 2020), a análise de redes sociais desponta como uma dessas possibilidades, que será abordada a seguir.

\section{ANÁLISE DE REDES SOCIAIS}

O ponto de partida para a análise de redes sociais no desempenho esportivo perpassa pela teoria dos grafos, que vem sendo utilizada para representar as interações em equipes esportivas, compreendidas como sistemas dinâmicos e complexos (Clemente, Martins, \& Mendes, 2015; Ribeiro et al., 2017). Advindo da matemática, um grafo, ou sociograma, é composto por arestas (i.e. ações) e vértices (i.e. atores das ações) (Borgatti et al., 2009). Ao se aplicar a teoria dos grafos para a representação das equipes esportivas, um grafo é a representação das interações que ocorreram no evento estudado (e.g. uma partida, uma situação ofensiva, uma atividade de treino). Uma das formas comumente utilizadas para compreender a interação dos jogadores na fase ofensiva refere-se à realização de passes bem-sucedidos, representado no grafo como arestas. Porém, embora mais comum, não necessariamente apenas os passes bem-sucedidos podem ser levados em consideração. Posteriormente será exemplificado outras possibilidades (e.g. passes malsucedidos). Os jogadores no grafo são representados pelos vértices entre as arestas (Clemente, Martins, \& Mendes, 2016).

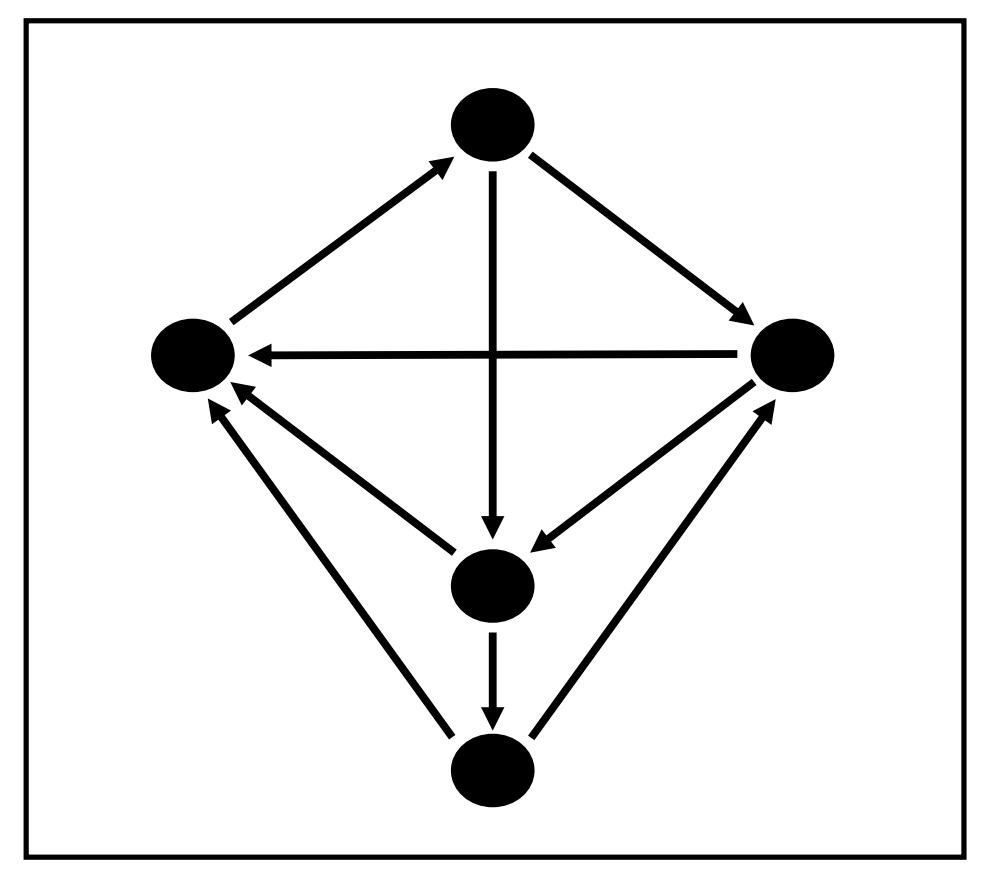

Figura 1. Representação das interações de um grafo ocorrentes em um evento. Os vértices (bolas pretas) representam os jogadores. As setas (arestas) correspondem às interações dos jogadores. Fonte: Os autores. 
Para a formação do grafo e do estudo das interações interpessoais, é necessária a construção de uma matriz de adjacência para que seja possível estruturar as interações (i.e. passes), os membros que fazem parte do grafo (i.e. jogadores) e o direcionamento das interações (i.e. de quem para quem) (Clemente, Martins, \& Mendes, 2015). Sendo assim, a figura $\underline{2}$ representa um grafo fictício das interações ocorridas em um evento de futsal, e a Tabela 1 corresponde a matriz de adjacência das interações desse evento. Após formado o grafo que se deseja estudar e sua matriz de adjacência, torna-se possível a aplicabilidade da análise de redes sociais aos esportes coletivos.

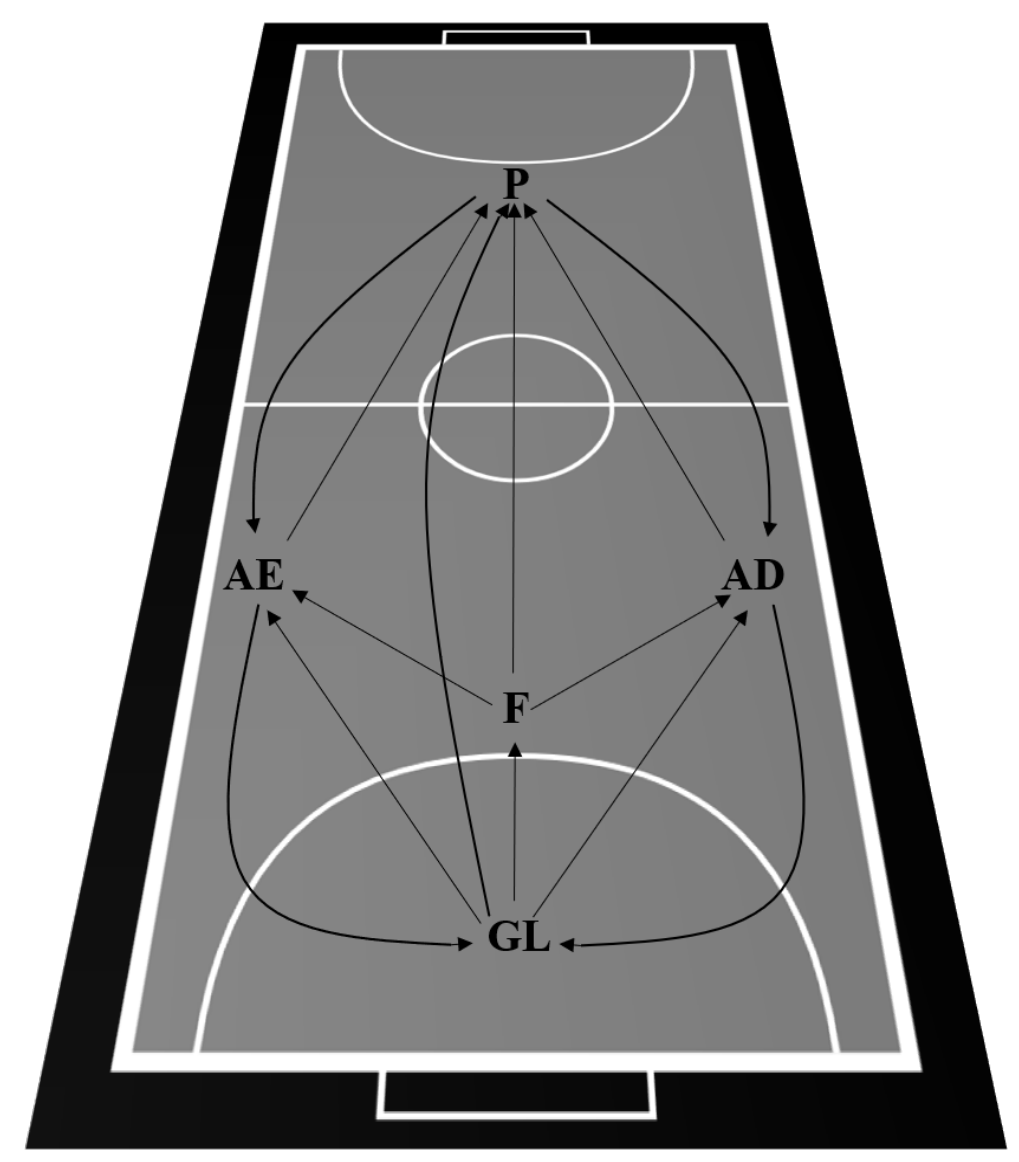

Figura 2. Representação gráfica das interações em um evento fictício de futsal. GL=goleiro, $F=$ fixo, $A E=$ ala esquerdo, $A D=$ ala direito, $P=$ =pivô. Fonte: Os autores. 
Tabela 1.

Matriz de adjacência referente as interações ocorridas em um evento fictício de futsal.

\begin{tabular}{|c|c|c|c|c|c|}
\hline- & $\mathbf{G L}$ & $\mathbf{F}$ & $\mathbf{A E}$ & $\mathbf{A D}$ & $\mathbf{P}$ \\
\hline $\mathbf{G L}$ & - & 1 & 1 & 1 & 1 \\
\hline $\mathbf{F}$ & 0 & - & 1 & 1 & 1 \\
\hline $\mathbf{A E}$ & 1 & 0 & - & 0 & 1 \\
\hline $\mathbf{A D}$ & 0 & 0 & 0 & - & 1 \\
\hline $\mathbf{P}$ & 0 & 0 & 1 & 1 & - \\
\hline
\end{tabular}

Nota. $G L=$ goleiro, $F=$ fixo, $A E=$ ala esquerdo, $A D=$ ala direito, $P=$ pivô. Fonte: $O$ s autores.

Por meio da análise de redes sociais busca-se analisar as ações cooperativas de interação entre membros que constituem um grupo, uma vez que as interações e os aspectos de coesão e hierarquia nas equipes demonstram-se fortemente presentes em esportes coletivos (Clemente et al., 2014; Lusher et al., 2010). Sendo assim, o principal objetivo na análise de redes sociais é estudar as relações entre os jogadores para identificar possíveis padrões em um grupo, assim como o entendimento dos processos coordenativos coletivos que ajudam a compreender o desempenho esportivo (Clemente, Martins, \& Mendes, 2015). Assim, as equipes passam a ser analisadas como grupos sociais e não como sujeitos isolados. Com isso, diferencia-se das análises tradicionais, nas quais o principal foco está no sujeito (Clemente, Martins, \& Mendes, 2015), ou em análises notacionais que buscam apenas a acumulação da frequência dos eventos ocorridos (e.g. gols marcados, posse de bola, zonas de finalização) (Hughes \& Bartlett, 2002).

Além disso, a análise de redes sociais visa investigar padrões de interações bidirecionais do macro (i.e. a estrutura como um todo) ao micro (i.e. métricas individuais) (Clemente, Martins, \& Mendes, 2016; Ribeiro et al., 2017). Ao nível de microanálise, as métricas se voltam para variáveis individuais que permitem identificar a contribuição do jogador para as interações dentro do grafo e se baseiam na centralidade (i.e. número de ligações) (Clemente, Martins, \& Mendes, 2016). Sendo assim, as métricas individuais comumente utilizadas são: número de interações recebidas por um indivíduo (in-degree centrality), número de interações realizadas por um indivíduo (out-degree centrality), somatória das variáveis in-degree e out-degree (grau ponderado), a representação do quão perto o jogador está dos outros jogadores, sendo que um baixo valor dessa variável demonstra certa proximidade com os companheiros de equipe (closeness centrality), o valor do número de vezes que um jogador intermediou a ligação de outros dois (betweenness centrality), a identificação dos possíveis jogadores-chave que 
desempenham um papel crucial na organização das fases ofensivas (eigenvector centrality) (Castellano \& Echeazarra, 2019; Clemente, Martins, \& Mendes, 2016; Peña \& Touchette, 2012).

Com isso, ao nível de macroanálise, visa-se o estudo de variáveis coletivas, com o objetivo de estudar o processo de organização das equipes (Clemente, Martins, \& Mendes, 2016). Dessa forma, as métricas mais comuns são: descrição do nível geral de cooperação interpessoal mostrado por companheiros de equipe, logo, valores mais altos identificam uma melhor homogeneidade de interações entre jogadores de uma equipe (i.e. densidade) e a informação sobre a formação de subgrupos de jogadores que coordenam com mais frequência suas ações por meio de passes, sendo que, valores mais altos apontam a capacidade de formar subgrupos funcionais dentro das equipes (i.e. coeficiente de clustering) (Castellano \& Echeazarra, 2019; Clemente, Martins, \& Mendes, 2016; Peña \& Touchette, 2012). Na tabela 2 pode-se visualizar as métricas, suas definições e implicações para a análise. 
Tabela 2.

Descrição e implicações práticas das métricas da análise de redes sociais para a análise de desempenho

\begin{tabular}{llll}
\hline \multicolumn{1}{c}{ Métricas } & \multicolumn{1}{c}{ Conceito } & \multicolumn{1}{c}{ Implicação para a análise } \\
\hline In-degree centrality & $\begin{array}{l}\text { Número de interações } \\
\text { recebidas. }\end{array}$ & $\begin{array}{l}\text { Quantidade de passes que o atleta } \\
\text { recebeu. }\end{array}$ \\
Out-degree centrality & $\begin{array}{l}\text { Número de interações } \\
\text { realizadas. }\end{array}$ & $\begin{array}{l}\text { Quantidade de passes que o atleta } \\
\text { realizou. }\end{array}$ \\
Grau ponderado & $\begin{array}{l}\text { Somatória das métricas } \\
\text { in-degree e ou-degree }\end{array}$ & $\begin{array}{l}\text { Quantidade de interações em que o atleta } \\
\text { se envolveu. }\end{array}$ \\
Closeness centrality & $\begin{array}{l}\text { Representa o quão } \\
\text { próximo o vértice } \\
\text { encontra-se dos outros. }\end{array}$ & $\begin{array}{l}\text { Restántação do quão próximo o atleta } \\
\text { companheiros. Valores mais baixos } \\
\text { representam certa proximidade com os } \\
\text { companheiros de equipe. }\end{array}$
\end{tabular}

Beteweenness centrality Representação numérica das intermediações realizadas por um vértice O número de vezes que o atleta intermediou as interações entre dois companheiros. para outros dois.

Eigenvector centrality

Representação

da Torna-se possível identificar possíveis relevância do vértice jogadores-chave na organização para as interações do defensiva da equipe. grafo.

Densidade

Descrição no nível geral

Representação do nível de distribuição de interações de um das interações da equipe. Quanto maior o grafo. valor, maior a homogeneidade das interações que ocorrem na equipe.

Coeficiente de clustering Formação de possíveis Representação da formação de subgrupos subgrupos dentro do dentro da equipe, valores mais altos sugerem que a equipe possui alta capacidade de formar subgrupos que coordenam as ações ofensivas dentro da equipe.

Fonte: adaptado de Ribeiro et al. (2017). 


\section{IMPLICAÇÕES PRÁTICAS DA ANÁLISE DE REDES SOCIAIS NOS ESPORTES COLETIVOS}

Após a compreensão das métricas provenientes da análise de redes sociais, torna-se possível aplicá-las no contexto de análise de desempenho e fornecer informações relevantes para os treinadores. Iniciando pelas métricas individuais, são baseadas na variável centrality, que fornece informações sobre a relevância do jogador para as interações ocorridas na equipe (Ribeiro et al., 2017). Sendo assim, as métricas in-degree centrality e out-degree centrality são baseadas simplesmente no número de interações realizadas e recebidas, fornecendo um dado simples para a comissão técnica da frequência de passes que os jogadores realizaram ou receberam (Clemente, Martins, \& Mendes, 2016). Quanto menor for o valor de closeness centrality de um jogador, menor a distância entre ele e seus companheiros de equipe, o que aumenta a probabilidade de interação com a equipe ou participar ativamente nas situações ofensivas (Clemente, Martins, \& Mendes, 2016; Ribeiro et al., 2017). Um jogador com altos valores de betweenness centrality seria altamente responsável pela ligação entre seus companheiros de equipe, ou seja, um jogador com alto grau de responsabilidade na ligação entre diferentes setores da equipe (Borgatti, 2005; Borgatti et al., 2009; Clemente, Martins, \& Mendes, 2016), como por exemplo a ligação entre a defesa e o ataque no futebol, ou entre diferentes alas no futsal. O jogador que obtiver maior valor da métrica de eigenvector centrality, será o jogador que mais apresentou relevância nas interações ocorridas na equipe, dessa forma, baixos valores dessa métrica representa pouca contribuição nas interações de forma geral da equipe (Borgatti, 2005; Borgatti et al., 2009; Clemente, Martins, \& Mendes, 2016).

As métricas coletivas contribuem para o entendimento da dinâmica coletiva do jogo (Ribeiro et al., 2017). Por exemplo, os valores de densidade e coeficiente de clustering variam de 0 (falta de cooperação) a 1 (cooperação máxima) (Ribeiro et al., 2017). Uma equipe com valores próximos a 1, significa maior homogeneidade de interações dos jogadores e boa capacidade funcional de formar subgrupos. Por outro lado, uma equipe com valores próximos a 0 , significa maior heterogeneidade de interações dos jogadores e pouca capacidade funcional de formar subgrupos.

Além disso, é necessário que os critérios para análise sejam pré-definidos de acordo com o objetivo dos treinadores e/ou pesquisadores. Por exemplo, a matriz de adjacência pode ser elaborada a partir das interações contabilizadas por todos os passes (bem-sucedidos e malsucedidos), apenas por passes bem-sucedidos ou apenas por passes malsucedidos. Além disso, a situação em que os passes ocorreram também influenciarão na resposta que a análise fornecerá (i.e. todas as situações, situações que geraram oportunidades de finalização, situações que geraram progressão no terreno de jogo adversário).

Dessa forma, se a análise possui como finalidade estudar como a equipe gerencia a posse de bola de forma geral, todos os passes podem ser levados em consideração. Caso a análise tenha como objetivo compreender como ocorre a progressão da bola no terreno de jogo 
adversário, apenas passes para frente ou que quebrem linhas defensivas podem ser considerados. Porém, se a finalidade da análise for observar como a equipe gerencia os passes que geraram situações de finalização, apenas passes que criaram oportunidades de finalização deveram ser computados. Para finalizar, o objetivo também pode ser estudar as dificuldades e erros nas tentativas de progressão no terreno de jogo adversário, categorizando assim, apenas os passes errados (Mclean \& Salmon, 2019).

Com isso, é importante destacar que essas métricas devem ser analisadas dentro de um contexto e não de forma isolada. Sendo assim, torna-se possível identificar informações sobre as dinâmicas que regem as interações da equipe, e transpô-las para o ambiente de treino. Por exemplo, a comissão técnica de uma equipe identificou que possui baixa formação de subgrupos (i.e. baixos valores de coeficiente de clustering) e baixo valor de densidade dos grafos no decorrer dos jogos de sua equipe. Além disso, identificou que seus jogadores eram dependentes de apenas um jogador chave para que as interações ocorressem na equipe, ou seja, um jogador apenas com alto valor de eigenvector centrality. Possivelmente, tal situação representaria um problema para um jogo coletivo desta equipe, pela forte dependência de apenas um jogador. Dessa forma, uma possível solução seria propor durante as sessões de treino, situações em que a equipe seja estimulada à formação de subgrupos, como por exemplo a ligação entre diferentes setores específicos da equipe (e.g. setor defensivo esquerdo e setor ofensivo direito). Para isso, a comissão técnica poderia identificar jogadores que mais foram responsáveis por ligar outros dois companheiros de equipe, ou seja, que apresentaram maiores valores de betweenness centrality e os inserirem em situações que estimulem tais interações dos diferentes setores desejados. Além disso, nesse mesmo exemplo, regras que favoreçam um jogo coletivo poderiam ser aplicadas para estimular maior cooperação e maior homogeneidade de interações (densidade da equipe e valores altos de eingenvector centrality em mais jogadores), como por exemplo o limite de toques na bola.

Podemos destacar mais quatro implicações práticas relevantes da análise de redes sociais: i) a relevância das interações entre os jogadores que constituem a equipe; ii) o significado dos dados sustentados pelas interações sociais; iii) descrição dos padrões das interações por meio de imagens gráficas e; iv) a descrição entre os agentes que constituem o sistema e compõem as interações por meio de modelagem computacional e matemática (Ribeiro et al., 2017). Além disso, a aplicação da análise de redes sociais também pode ser utilizada para melhor compreensão das relações entre os espaços do campo/quadra. Por exemplo, se utilizar como "nó" os quadrantes do campo/quadra, é possível ter a informação da maior concentração de circulação da posse de bola em setores específicos do campo/quadra.

Observa-se na literatura estudos que investigaram métricas de análise de redes sociais nos esportes coletivos (e.g. futsal, futebol, basquetebol, handebol, voleibol). No futsal, as situações que resultaram em gols apresentaram maior objetividade e menores valores interativos 
do que as situações que não resultaram em gols (i.e. menores valores de densidade e betweenness centrality) (Vieira et al., 2015). Os autores sugerem que, possivelmente, as equipes que souberam explorar melhor as condições impostas pelo adversário e com menor número de interações tenderam a obter maior êxito nas situações ofensivas. No futebol, a manipulação da quantidade de jogadores presentes na atividade (i.e. 4 vs. 3,3 vs. $3+2$ e 3 vs. 3 ) alteraram os padrões de interação, com maiores valores de densidade e coeficiente de clustering durante o formato 4 vs. 3 (Moreira et al., 2017). Além disso, um estudo recente mostra que as métricas individuais e coletivas da análise de redes sociais podem ser aplicadas em contexto de treinamento como indicadores de ajuste das tarefas ao nível de desempenho tático dos jogadores, de forma a ajustar o processo de ensino e treinamento as dinâmicas intrínsecas dos jogadores (Machado et al., 2020).

Além disso, durante os jogos de futebol da Copa do Mundo da FIFA de 2018, não foram encontradas diferenças em variáveis de macroanálise (i.e. densidade e coeficiente de clustering) em função dos resultados momentâneos (i.e. ganhando vs. empatando vs. perdendo) (Moreira et al., 2019). Porém, foram apontadas diferenças em variáveis de microanálise (i.e. métricas individuais), apresentando alterações importantes nas ações individuais de acordo com o resultado momentâneo, sugerindo um estilo de jogo mais direto em situações de vitória e um estilo de jogo mais indireto (posse de bola) em situações de derrota (Moreira et al., 2019). Ainda durante a Copa do Mundo de 2018, maiores valores de coeficiente de clustering foram verificados quando as equipes ganharam comparado com as derrotas (Moreira et al., 2019). Por exemplo, a França (campeã mundial) apresentou uma média de 0,746 unidades arbitrárias de coeficientes de clustering, enquanto o Panamá (uma das equipes com menor classificação) apresentou uma média de 0,644 unidades arbitrárias. Esses resultados sugerem que os jogadores franceses apresentaram maior cooperação. Em outro estudo, também foi relatado que as equipes que atingiram as fases finais da Copa do Mundo de 2014 apresentaram altos valores de coeficientes de clustering, o que pode levar a um alto nível de eficácia ofensiva (Clemente, Martins, Kalamaras, et al., 2015). Além disso, um estudo de larga escala (283.529 passes registrados em 760 partidas de futebol) na Premier League inglesa demonstrou que os gols marcados estavam associados às métricas de densidade e centralização (Grund, 2012). Ou seja, altos níveis de densidade levaram a um aumento do desempenho da equipe, enquanto menores valores de densidade foram associados a uma diminuição no desempenho da equipe.

Por outro lado, estudos com foco em fases ofensivas e gols marcados sugerem que a densidade reduzida pode ajudar a alcançar um melhor desempenho da equipe (Mclean et al., 2018; Pina et al., 2017). Portanto, sugerimos cautela nas relações das métricas de análise de redes sociais e o resultado final das partidas. Coletivamente, esses resultados sugerem que as métricas individuais e globais mostram mais potencial para fornecer informações práticas sobre como as equipes organizam seus processos ofensivos do que informações relacionadas ao que 
discrimina entre equipes bem ou malsucedidas, por exemplo, adotando um estilo de jogo mais direto, por meio de um contra-ataque, ou um estilo mais indireto, por meio de um ataque posicional. As equipes de sucesso podem optar por estilos de jogo distintos de acordo com os jogadores à sua disposição, a filosofia do treinador/clube, a cultura local, entre outros fatores. Portanto, a variedade de modelos de jogos bem-sucedidos pode resultar em alta variabilidade das métricas individuais e globais obtidas pela análise de redes sociais (Aquino et al., 2019) No basquetebol (Fewell et al., 2012), a utilização das métricas individuais de centralidade e a métrica global de coeficiente de clustering mostraram-se eficazes para distinguir as estratégias das equipes da NBA (National Basketball Association), edição de 2010, quanto suas interações interpessoais. Além disso, os autores citados sugerem que equipes que contenham lideranças mistas quanto ao papel interativo na quadra se mostraram mais eficientes ofensivamente do que equipes que dependem fortemente de uma liderança centralizada ou de equipes com lideranças altamente descentralizadas, o que pode estar relacionado com valores mais altos de eingenvector.

Um estudo realizado por Korte e Lames (2018), com o objetivo de caracterizar diferentes esportes coletivos (i.e. futebol, handebol e basquetebol) por meio da análise de redes sociais, mostrou que o handebol possui um maior desequilíbrio na distribuição de suas interações em comparação com o futebol e basquetebol. Além disso, os autores sugeriram que as posições de jogo em cada esporte possuem forte influência nos valores das métricas de redes sociais. No futebol, o meio-campista defensivo (popularmente denominado de "volante") apresentou os maiores valores para as métricas individuais de redes sociais. Já no basquetebol, o armador (point guard) foi quem apresentou maior influência nas interações das equipes. No handebol, o armador central (center) foi a posição que apresentou maior importância na distribuição das interações das equipes. As métricas utilizadas nesse estudo foram in-degree, out-degree, betweenness centrality, e closeness centrality (Korte \& Lames, 2018).

Um estudo recente buscou caracterizar o handebol por meio da ferramenta de análise de redes sociais utilizando 22 partidas do Campeonato Europeu de Handebol Masculino de 2018 (Korte \& Lames, 2019). Os autores verificaram diferenças significativas de acordo com as posições de jogo durante as fases ofensivas. As posições left back, right back e center foram as maiores responsáveis pelas interações ofensivas, independente do sistema de jogo utilizado pelas equipes, o que certamente se relaciona com a função desses jogadores (armadores). Além disso, os autores verificaram maior interação quando a equipe adotava o sistema de jogo 7 vs. 6 (i.e. sem goleiro), e as posições right wing e left wing foram mais acionadas quando a equipe adversária estava com um jogador a menos (i.e 6 vs. 5), o que demonstra maiores possibilidades de explorar as lacunas laterais por conta da vantagem numérica da equipe com posse de bola. No voleibol, pesquisadores mostram que a métrica de eigenvector possui a maior utilidade nas 
análises das partidas oficiais, já que representa mais do que uma simples conexão entre os jogadores (Santos, 2016).

É importante destacar que outras abordagens de análise de redes sociais têm sido propostas no âmbito esportivo, como a hypernetworks (Ribeiro et al., 2019; 2020). Nesse caso, estende-se o conceito descrito na formação dos grafos por díades (i.e. ligações entre dois vértices, ou seja, interações entre dois jogadores) para a formação de hipergrafos, sendo compreendido como a generalização de um grafo. Com isso, as interações podem considerar quaisquer quantidades de vértices (i.e. formação de tríades, tétrades, etc.) (Ribeiro et al., 2019; 2020).

\section{CONSIDERAÇÕES FINAIS}

É imprescindível a compreensão de que as possibilidades de ação (i.e. affordances) possuem papel fundamental na compreensão da relação entre a abordagem ecológica e a utilização da análise de redes sociais. O processo de interação/coordenação de uma equipe é orientado por meio da percepção das possibilidades de ação e de como ocorrem as relações mútuas jogador-jogador, jogador-equipe, jogador-ambiente e equipe-ambiente (Silva et al., 2013). Para isso, essas interações podem ser expressas por meio das métricas individuais e coletivas obtidas pela análise de redes sociais, que são sustentadas por sinergias interpessoais dos jogadores (Silva et al., 2013).

No entanto, é importante destacar algumas limitações da análise de redes sociais, tal como o tempo de análise. Contudo, alguns softwares como o uPATO contribuem para reduzir este tempo de análise (Clemente, Martins, Mendes, et al., 2016). Outra limitação importante a ser destacada é o fato do jogo não se resumir a passes. Logo, apesar da análise de redes sociais ter a possibilidade de considerar importantes eventos na partida (e.g. gols, ataques, finalizações, desarmes, interceptações), esta abordagem de análise, geralmente, não inclui nas interações estes eventos. Para isso, sugere-se a utilização de métodos que descrevam as ações ocorridas e suas frequências durante as partidas analisadas (Travassos et al., 2013). Portanto, a análise de redes sociais pode complementar, não substituir, outros métodos de análise de desempenho (e.g. análise notacional, análise sequencial, coordenação interpessoal por meio de dados posicionais). 


\section{REFERÊNCIAS}

Aquino, R., Machado, J. C., Clemente, F. M., Moreira, G., Gonçalves, L. G., Melli-Neto, B., Ferrari, J. V., Palucci, L. H. ... Carling, C. (2019). Comparisons of ball possession, match running performance, player prominence and team network properties according to match outcome and playing formation during the 2018 FIFA World Cup. International Journal of Performance Analysis in Sport, 19(6), 1026-1037. doi: https://doi.org/10.1080/24748668.2019.1689753

Araújo, D. \& Davids, K. (2009). Ecological approaches to cognition and action in sport and exercise: Ask not only what you do, but where you do it. International Journal of Sport Psychology 40(1), 5-239. Recuperado de https://www.researchgate.net/profile/Duarte Araujo/publication/38183786 Ecological app roaches to cognition and action in sport and exercise Ask not only what you do b ut where you do it/links/0912f50aff778383cd000000/Ecological-approaches-tocognition-and-action-in-sport-and-exercise-Ask-not-only-what-you-do-but-where-you-doit.pdf\#page $=10$

Araújo, D. \& Davids, K. (2011). What Exactly is Acquired During Skill Acquisition? Journal of Consciousness Studies 18(3-4) 7-23. Recuperado de https://www.ingentaconnect.com/content/imp/jcs/2011/00000018/f0020003/art00001

Barreira, D. (2014). Tendências evolutivas da dinâmica tática em Futebol de alto rendimento. Estudo da fase ofensiva nos Campeonatos da Europa e do Mundo, entre 1982 e 2010 (Tese de doutorado). Recuperado de https://repositorio-aberto.up.pt/handle/10216/71273

Borgatti, S. P. (2005). Centrality and network flow. Social Networks, 27(1), 55-71. doi: https://doi.org/10.1016/j.socnet.2004.11.008

Borgatti, S. P., Mehra, A., Brass, D. J. \& Labianca, G. (2009). Network Analysis in the Social Sciences. American Association for the Advancement of Science, 323(5916), 892-895. doi: https://doi.org/10.1126/science.1165821

Carling, C., Williams, A. M. \& Reilly, T. (2005). Handbook of soccer match analysis. A systematic approach to improving performance. London: Routledge. Recuperado de https://www.taylorfrancis.com/books/mono/10.4324/9780203448625/handbook-soccermatch-analysis-christopher-carling-mark-williams-thomas-reilly

Castellano, J. \& Echeazarra, I. (2019). Network-based centrality measures and physical demands in football regarding player position: Is there a connection? A preliminary study. Journal of Sports Sciences, 37(23), 2631-2638. doi: https://doi.org/10.1080/02640414.2019.1589919

Clemente, F. M., Santos, M., Lourenço, F. M. \& Sousa, R. (2014). Using network metrics to investigate football team players' connections: A pilot study. Motriz: Revista de Educação Física, 20(3), 262-271. doi: https://doi.org/10.1590/S1980-65742014000300004 
Clemente, F. M., Lourenço, F. M. \& Sousa, R. (2015). Social network analysis: Um ensaio sobre a aplicabilidade na análise de cooperação em contextos esportivos. Conexões, 13(3), 175194. doi: https://doi.org/10.20396/conex.v13i3.8640877

Clemente, F. M., Lourenço, F. M. \& Sousa, R. (2016). Social Network Analysis Applied to Team Sports Analysis. Manhattan, United States: Springer International Publishing. doi: https://doi.org/10.1007/978-3-319-25855-3

Clemente, F. M., Lourenço, F. M., Kalamaras, D., Del Wong, P. \& Sousa, R. (2015). General network analysis of national soccer teams in FIFA World Cup 2014. International Journal of Performance Analysis in Sport, 15(1), 80-96. doi: https://doi.org/10.1080/24748668.2015.11868778

Clemente, F. M., Lourenço, F. M., Sousa, R. \& Silva, F. (2016). Social network measures to match analysis in soccer: A survey. Journal of Physical Education and Sport, 16(03), 823-830. doi: https://doi.org/10.7752/jpes.2016.03130

Duarte, R., Araújo, D., Correia, V. \& Davids, K. (2012). Sports teams as superorganisms. Implications of sociobiological models of behaviour for research and practice in team sports performance analysis. Sport Medicine, 42(8), 633-642. doi: https://link.springer.com/article/10.1007/BF03262285

Edelman, G. M. \& Gally, J. A. (2001). Degeneracy and complexity in biological systems. Proceedings of the National Academy of Sciences, 98(24), 13763-13768. doi: https://doi.org/10.1073/pnas.231499798

Ettekal, A. \& Mahoney, J. L. (2017). Ecological Systems Theory. In K. Peppler (Ed.), The SAGE Encyclopedia of Out-of- School Learning (pp. 239-241). United States: SAGE Publications, Inc. doi: https://doi.org/10.4135/9781483385198

Fewell, J. H., Armbruster, D., Ingraham, J., Petersen, A. \& Waters, J. S. (2012). Basketball Teams as Strategic Networks. PLoS ONE, 7(11), e47445. doi: https://doi.org/10.1371/journal.pone.0047445

Galatti, L. R., Silva, R., Scaglia, A. J., Rodrigues, R. \& Montero, A. (2014). Pedagogia do Esporte: Tensão na ciência e o ensino dos Jogos Esportivos Coletivos. Revista da Educação Física/UEM, 25(1), 153-162. doi: https://doi.org/10.4025/reveducfis.v25i1.21088

Garganta, J. (1995). Para uma teoria dos jogos coletivos desportivos. O ensino de jogos desportivos. Porto, Portugal: Universidade do Porto.

Garganta, J. (2001). A análise da performance nos jogos desportivos. Revisão acerca da análise do jogo. Revista Portuguesa de Ciências do Desporto, 1(1), 57-64. Recuperado de https://rpcd.fade.up.pt/ arquivo/artigos soltos/vol.1 nr.1/08.pdf

Gibson, J. J. (1979). The ecological approach to visual perception. Psychology Press.

Gibson, J. J. (1983). The Senses Considered as Perceptual Systems. London: UNWIN LTD. Recuperado 
https://monoskop.org/images/d/df/Gibson James J The Sense Considered as Percept ual Systems 1966.pdf

Groom, R., Cushion, C. \& Nelson, L. (2011). The Delivery of Video-Based Performance Analysis by England Youth Soccer Coaches: Towards a Grounded Theory. Journal of Applied Sport Psychology, 23(1), 16-32. doi: https://doi.org/10.1080/10413200.2010.511422

Grund, T. U. (2012). Network structure and team performance: The case of English Premier League soccer teams. Social Networks, 34(4), 682-690. doi: https://doi.org/10.1016/j.socnet.2012.08.004

Hatfield, B. \& Kerick, S. (2007). Handbook of Sport Psychology. In G. Tenenbaum \& R. Eklund $(E d), \quad\left(3^{\text {rd }}\right.$ ed.). New Jersey: John Wiley \& Sons, Inc. Recuperado de https://www.researchgate.net/publication/294693225

Hughes, M. D. \& Bartlett, R. M. (2002). The use of performance indicators in performance analysis. Journal of Sports Sciences, 20(10), 739-754. doi: https://doi.org/10.1080/026404102320675602

Ingold, T. (2002). The Perception of the Environment: Essays on Livelihood, Dwelling and Skill. London: Routledge. doi: https://doi.org/10.4324/9780203466025

Korte, F. \& Lames, M. (2018). Characterizing different team sports using network analysis. Current Issues in Sport Science (CISS), 3, 1-11. doi: https://doi.org/10.15203/CISS 2018.005

Korte, F. \& Lames, M. (2019). Passing Network Analysis of Positional Attack Formations in Handball. Journal of Human Kinetics, 70(1), 209-221. doi: https://doi.org/10.2478/hukin2019-0044

Low, B., Coutinho, D., Gonçalves, B., Rein, R., Memmert, D. \& Sampaio, J. (2020). A Systematic Review of Collective Tactical Behaviours in Football Using Positional Data. Sports Medicine, 50, 343-385. doi: https://doi.org/10.1007/s40279-019-01194-7

Lusher, D., Robins, G. \& Kremer, P. (2010). The Application of Social Network Analysis to Team Sports. Measurement in Physical Education and Exercise Science, 14(4), 211-224. doi: https://doi.org/10.1080/1091367X.2010.495559

Machado, J., Aquino, R., Góes, A., Bosco, J., Barreira, D., Travassos, B., Ibanez, S. \& Scaglia, A. (2020). Macro and micro network metrics as indicators of training tasks adjustment to players' skills level. International Journal of Sports Science \& Coaching, 16(3), 1-9. doi: https://doi.org/10.1177/1747954120979561

Machado, J., Barreira, D., Galatti, L., Chow, J., Garganta, J. \& Scaglia, A. (2019). Enhancing learning in the context of Street football: A case for Nonlinear Pedagogy. Physical Education and Sport Pedagogy, 24(2), 176-189. doi: https://doi.org/10.1080/17408989.2018.1552674 
Mclean, S. \& Salmon, P. M. (2019). The weakest link: A novel use of network analysis for the broken passing links in football. Science and Medicine in Football, 3(3), 255-258. doi: https://doi.org/10.1080/24733938.2018.1562277

Mclean, S., Salmon, P., Gorman, A., Stevens, N. \& Solomon, C. (2018). A social network analysis of the goal scoring passing networks of the 2016 European Football Championships. Human Movement Science, 400-408. doi:_https://doi.org/10.1016/j.humov.2017.10.001

Menezes, R. (2012). Contribuições da concepção dos fenômenos complexos para o ensino dos esportes coletivos. Motriz: Revista de Educação Física, 18(1), 34-41. doi: https://doi.org/10.1590/S1980-65742012000100004

Menezes, R., Rodrigues, R. \& Nunomura, M. (2014). Especialização esportiva precoce e o ensino dos jogos coletivos de invasão. Movimento: Revista de Educação Física Da UFRGS, 20(1), 351-373. doi: https://doi.org/10.22456/1982-8918.40200

Moreira, G, Barbosa, B., Teles, S., Brito, R., Clemente, F. \& Pereira, A. (2019). Influence of Match Status on Players' Prominence and Teams' Network Properties During 2018 FIFA World Cup. Frontiers in Psychology, 10, 1-8. doi: https://doi.org/10.3389/fpsyg.2019.00695

Moreira, G., Clemente, F., Perez, J., Greco, P. \& Pereira, A. (2017). Network analysis in smallsided and conditioned soccer games: the influence of additional players and playing position. Kinesiology, 49(2), 185-193. doi: https://doi.org/10.26582/k.49.2.8

O'Donoghue, P. (2009). Research Methods for Sports Performance Analysis. London: Routledge. doi: https://doi.org/10.4324/9780203878309

Passos, P., Araújo, D., Davids, K., Gouveia, L., Serpa, S., Milho, J. \& Fonseca, S. (2009). Interpersonal Pattern Dynamics and Adaptive Behavior in Multiagent Neurobiological Systems: Conceptual Model and Data. Journal of Motor Behavior, 41(5), 445-459. doi: https://doi.org/10.3200/35-08-061

Peña, J. \& Touchette, H. (2012). A network theory analysis of football strategies. Recuperado de https://arxiv.org/abs/1206.6904

Pina, T., Paulo, A. \& Araújo, D. (2017). Network Characteristics of Successful Performance in Association Football. A Study on the UEFA Champions League. Frontiers in Psychology, 8 , 1-7. doi: https://doi.org/10.3389/fpsyg.2017.01173

Ribeiro, J., Garganta, J., Davids, K. \& Barreira, D. (2020). A multilevel hypernetworks approach to assess coordination and communication in player interactions in sports teams as coevolutionary networks. Brazilian Journal of Motor Behavior, 14(5), 167-170. doi: https://doi.org/10.20338/bjmb.v14i5.216

Ribeiro, J., Lopes, R., Silva, P., Araújo, D., Barreira, D., Davids, K., Ramos, J., Maia, J. \& Garganta, J. (2019). A multilevel hypernetworks approach to capture meso-level 
synchronisation processes in football. Journal of Sports Sciences, 38(5), 494-502. doi: https://doi.org/10.1080/02640414.2019.1707399

Ribeiro, J., Silva, P., Duarte, R., Davids, K. \& Garganta, J. (2017). Team Sports Performance Analysed Through the Lens of Social Network Theory: Implications for Research and Practice. Sports Medicine, 47(9), 1689-1696. doi: https://doi.org/10.1007/s40279-017$\underline{0695-1}$

Santos, M. (2016). Mapeamento sistémico do jogo de voleibol feminino no alto nível através da análise de redes sociais (Dissertação de Mestrado). Universidade do Porto, Portugal. Recuperado de https://hdl.handle.net/10216/87885

Scaglia, A., Reverdito, R. \& Galatti, L. (2014). A contribuição da Pedagogia do Esporte na escola: Tensões e reflexões metodológicas. Em A. Marinho, J. V. Dos Nascimento \& A. A. B. Oliveira (Org.), Legados do esporte brasileiro (pp. 45-86). Brasil: UDESC. Recuperado de https://www.researchgate.net/publication/303541232 A contribuicao da Pedagogia do Esporte na escola tensoes e reflexoes metodologicas

Scott, J. (1995). Dynamic Patterns: The self-organization of brains and behaviour. Massachusetts, United States: The MIT Press. Recuperado de https://www.researchgate.net/publication/224982322 Dynamic Patterns The SelfOrganization of Brain and Behavior

Silva, P., Garganta, J., Araújo, D., Davids, K. \& Aguiar, P. (2013). Shared Knowledge or Shared Affordances? Insights from an Ecological Dynamics Approach to Team Coordination in Sports. Sports Medicine, 43(9), 765-772. doi: https://doi.org/10.1007/s40279-013-0070-9

Teodorescu, L. (1984). Problemas de teoria e metodologia nos jogos desportivos. Livros Horizonte.

Travassos, B., Araújo, D., Vilar, L. \& McGarry, T. (2011). Interpersonal coordination and ball dynamics in futsal (indoor football). Human Movement Science, 30(6), 1245-1259. doi: https://doi.org/10.1016/j.humov.2011.04.003

Travassos, B., Davids, K., Araújo, D. \& Esteves, T. (2013). Performance analysis in team sports: Advances from an Ecological Dynamics approach. International Journal of Performance Analysis in Sport, 13(1), 83-95. doi: https://doi.org/10.1080/24748668.2013.11868633

Travassos, B., Duarte, R., Vilar, L., Davids, K. \& Araújo, D. (2012). Practice task design in team sports: Representativeness enhanced by increasing opportunities for action. Journal of Sports Sciences, 30(13), 1447-1454. doi: https://doi.org/10.1080/02640414.2012.712716

Trevarthen, C. (1986). Development of Intersubjective Motor Control in Infants. In M. G. Wade \& H. T. A. Whiting (Ed.), Motor Development in Children: Aspects of Coordination and Control (pp. 209-261). Dordrecht: Martinus Nijhof. Recuperado de https://www.researchgate.net/publication/284613845 Development of Intersubjective M otor Control in Infants 
Vieira, A., Travassos, B., Cabo, M., Guerra, A. \& Esteves, P. (2015). Análise dos padrões de jogo ofensivo de seleções nacionais de futsal. Egitania Sciencia. Recuperado de https://www.researchgate.net/profile/Pedro Esteves2/publication/294841296 ANALISE DOS PADROES DE JOGO OFENSIVO DE SELECOES NACIONAIS DE FUTSAL/li nks/56c443dc08aea564e304a289.pdf

Vilar, L., Araújo, D., Davids, K. \& Button, C. (2012). The Role of Ecological Dynamics in Analysing Performance in Team Sports. Sports Medicine, 42(1), 1-10. doi: https://doi.org/10.2165/11596520-000000000-00000

Woods, C., Rudd, J., Robertson, S. \& Davids, K. (2020). Wayfinding: How Ecological Perspectives of Navigating Dynamic Environments Can Enrich Our Understanding of the Learner and the Learning Process in Sport. Sports Medicine - Open, 6(51). doi: https://doi.org/10.1186/s40798-020-00280-9 